\title{
Dynamic Pricing of Preemptive Service for Elastic Demand
}

\author{
Aylin Turhan, Murat Alanyali and David Starobinski
}

\begin{abstract}
We consider a service provider that accommodates two classes of users: primary users (PUs) and secondary users (SUs). SU demand is elastic to price whereas PU demand is inelastic. When a PU arrives to the system and finds all channels busy, it preempts an SU unless there are no SUs in the system. Call durations are exponentially distributed and their means are identical. We study the optimal pricing policy of SUs by using dynamic programming to maximize the total expected discounted profit over finite and infinite horizons, and the average profit. Our main contribution is to show that although the system is modeled as a two-dimensional Markov chain, the optimal pricing policy depends only on the total number of users in the system (PUs and SUs), i.e. the total occupancy. We also demonstrate that optimal prices are increasing with the total occupancy. Finally, we describe applications of these results to the special case of admission control and show that the optimal pricing policy structure of the original system is not preserved for systems with elastic PUs.
\end{abstract}

\section{INTRODUCTION}

Commercially available wireless spectrum has become drastically scarce because of the increasing use of wireless devices, such as smartphones and tablets. According to a recent study conducted on $2 \mathrm{G}, 3 \mathrm{G}$ and $4 \mathrm{G}$ networks by Ericsson, mobile data traffic will increase tenfold by 2016 and there will be 5 billion subscribers by then [1]. Although the market is in desperate need of additional spectrum, studies show that the spectrum allocated to license holders is often underutilized in space and time [2]. To improve spectrum utilization, cognitive radio (CR) technologies enable smart use of the spectrum through opportunistic spectrum hand-off and secondary market usage.

In CR systems, there are two classes of users: primary users (PUs) which have permanent license to access the spectrum and secondary users (SUs) which are temporarily accepted if the system is underutilized. A service provider (SP) serving both PUs and SUs must maximize its profit by attracting as many SUs as possible while ensuring that the performance perceived by PUs is not affected by the presence of SUs. We consider the PUs as higher priority users whereas the SUs are lower priority users. We use a preemption mechanism that aborts an SU call when a $\mathrm{PU}$ needs to make a call and there are no idle channels. As a practical application of preemption, FCC Block D at 700 $\mathrm{MHz}$ employs such a termination model where public safety services are the PUs and commercial services are the SUs [3].

This work was supported, in part, by the US National Science Foundation under grant CCF-0964652.

A. Turhan, M. Alanyali and D. Starobinski are with the Department of Electrical and Computer Engineering, Boston University, Boston, MA 02215, USA \{turhan, alanyali, staro\}@bu.edu
Our motivation is to characterize the structure of the optimal pricing policy in a preemptive system with inelastic PUs and elastic SUs. PUs have a constant demand whereas SU demand depends on the price advertised. A pricing policy enforces the prices advertised to the SUs. The rewards are collected upon the arrival of SUs and there is a cost per preempted SU.

In this work, we formulate a finite horizon discounted return dynamic programming (DP) problem to determine the optimal pricing policy that maximizes the profit. Then, we demonstrate that the value of an additional SU in the system depends only on the total occupancy, i.e. the total number of users in the system. Our main result is proving that the optimal pricing policy of SUs depends only on the total number of users (PUs and SUs) in the system for both infinite horizon and finite horizon profits. This property provides a simple and efficient way to determine the optimal pricing policy. Next, we establish a relationship between the optimal pricing policy and the total occupancy. We provide an average return DP formulation of the system using the total occupancy and demonstrate that relative rewards are decreasing and concave in total occupancy. Using these results, we deduce that the optimal prices increase with the total occupancy. Lastly, we discuss the application of our results to the special case of admission control. We also present a numerical counter-example to obtain the optimal pricing policy of a variant system with elastic PUs.

\section{RELATED WORK}

In this section, we present a literature review under three main categories: congestion-dependent pricing, dynamic control of networks and preemption.

Paschalidis and Tsitlikis [4] analyze congestion-dependent pricing of a multi-class network, where all classes have exponentially distributed call durations with different means and elastic arrival rates. Mutlu et al. [5] investigate the optimal dynamic pricing policy of a system consisting of inelastic PUs and elastic SUs with identical mean call durations. Gans and Savin [6] characterize a system consisting of two types of users within the context of a rental management problem which resemble the PUs and SUs in our model. Dube et al. [7] study the dynamic pricing of a single server system with identical and parallel queues.

The work of Miller [8] stands out as a seminal work in the field of admission control by considering a multi-class and multi-server queueing system. Ramjee et al. [9] study the two class version of the model of Miller. Ormeci et al. [10] study a two class loss system where the classes have different mean service rates. They deduce that the joint optimal admission 
control policy of two classes is of threshold type and depends on the number of users of each class.

The literature we have mentioned so far considers systems where users are only throttled upon arrival. The work after this point uses preemption. One of the earliest works on preemption is the work of Helly [11], which proposes approaches on the control of two class traffic with different priorities and limited capacity. Garay and Gopal [12] investigate the use of preemption control in high speed networks and analyze call preemption. Next, $\mathrm{Xu}$ and Shanthikumar [13] examine a first-come first-served non-identical multiserver system and determine its optimal admission control policy using duality and preemption. Brouns and van der Wal [14] study admission and preemption control of a two class single server queue with identical service rates. Brouns [15] extends these results to a multi-server system where there are no preemption costs. Finally, Ulukus et al. [16] consider the admission and preemption controls of a system with two classes, non-identical service rates and different priorities.

Our work differs from the preceding work, since we study pricing control in a preemptive system. Previous work on network control or pricing does not consider preemption, whereas previous work that utilizes preemption considers only preemption and/or admission control.

\section{MODEL DESCRIPTION}

In this section, we describe our model and statistical assumptions. We assume that there are $C$ identical and parallel channels which are allocated for the use of the calls of users of two classes: PUs and SUs.

We assume that each call requests the same amount of bandwidth corresponding to a single channel. PUs have preemptive priority over the SUs. In our case, a channel is allocated to a higher priority call even if a lower priority call is in progress. When the lower priority call is preempted, it is withdrawn from the system permanently.

Regardless of the class type, call durations are independent and exponentially distributed with mean $\mu^{-1}$ unless terminated prematurely. We model our system as a finite state two-dimensional (2D) continuous-time MDP. The rest of the system description is as follows:

States: The state of the system is in the form $(x, y)$ where $x \geq 0$ is the number of PU calls in the system and $y \geq 0$ is the number of SU calls in the system.

Rewards and costs: $u(x, y)$ is the reward per SU call at state $(x, y)$. The reward is collected upon arrival. A pricing policy is the set of rules which determines the price advertised by the SP at any given time, depending on the current state [4]. We denote the pricing policy as $\mathbf{u}$, and it is defined for the states within the range of the capacity limit $C$, i.e. $0 \leq x+y<C$. The prices at each state are chosen from an interval $\mathbb{U}=\left[0, u_{\max }\right]$ where a definition of $u_{\max }$ is provided below. We discretize $\mathbb{U}$ with a step size $\Delta u$ in order to obtain a finite control space. Then, the number of possible prices becomes $|\mathbb{U}|=\left\lfloor u_{\max } / \Delta u\right\rfloor+1$. From now on, we will use the discrete version of $\mathbb{U}$. If a PU call arrives and finds all the channels busy, then the system preempts an SU call exists any. The preemption mechanism is active only when all channels are busy. Whenever an SU call is preempted, the SP pays a cost $K>u_{\max }$. A call is blocked only if an arriving user finds all channels busy and preemption is not possible. For PUs, this corresponds to the case when all the calls in the system belong to PUs. For an incoming SU to be blocked, it is sufficient to have all $C$ channels busy. A blocked call receives a busy signal and is dropped. Blocking calls of any class is free of charge.

Arrival rates: $\mathrm{PU}$ calls arrive according to a Poisson process with a constant rate $\lambda_{1}>0$. SU calls, however, arrive according to a Poisson process and pay a fee $u(x, y)$ upon arrival when the state is $(x, y)$. The average arrival rate of SUs at state $(x, y)$ is related to the price $u(x, y)$ via a demand function $\lambda_{2}(u(x, y)) \geq 0$. We will use the following assumptions in all of our formulations:

Assumption 1: There exists a price $u_{\max }$ for which $\lambda_{2}(u(x, y))=0$ when $u(x, y) \geq u_{\max }$.

Assumption 2: $\lambda_{2}(u(x, y))$ is a strictly decreasing differentiable function of $u(x, y)$ over the interval $\left[0, u_{\max }\right]$.

Assumption 2 implies that the maximum possible arrival rate of SUs corresponds to the lowest possible price, i.e. $\lambda_{2, \max }=\lambda_{2}(0)$.

The objective of the SP is to maximize the average profit collected from SUs per unit time. The corresponding optimal pricing policy is denoted $\mathbf{u}^{*}$.

\section{ANALYSIS AND CHARACTERIZATION OF THE OPTIMAL PRICING POLICY}

In this section, we derive the average profit rate of SUs given a policy $\mathbf{u}$. Then, we present a finite horizon discounted return maximization problem formulation to compute the optimal pricing policy. Afterwards, we determine the structure of the optimal pricing policy that maximizes the discounted profit and extend our findings to the infinite horizon.

\section{A. Formulation of the Profit Maximization Problem}

In this section, we first introduce state space definitions and then develop a formula to calculate the average profit rate collected from SUs.

We start by defining state spaces. The entire state space is denoted as $S=\{(x, y) \mid x+y \leq C, \forall x, y \geq 0\}$. Let $S_{1} \subset S$ be the sub-space of states where all the channels are busy and at least one SU call is present in the system. According to our system description, $S_{1}$ denotes the states at which an SU can be preempted and is formally defined as $S_{1}=\{(x, y) \mid x+y=C, \forall x \geq 0, \forall y>0\}$. Lastly, we define $S_{2} \subset S$ which corresponds to all states where an SU arrival may enter the system, i.e. $S_{2}=$ $\{(x, y) \mid x+y<C, \forall x, y \geq 0\}$. We denote $\pi_{\mathbf{u}}(x, y)$ to be the steady state probability that the system is in state $(x, y)$ under the pricing policy $\mathbf{u}$. Note that $\mathbf{u}$ represents an arbitrary pricing policy that may not be necessarily optimal. The average profit rate under policy $\mathbf{u}$, is expressed as follows:

$$
J_{\mathbf{u}}=\sum_{(x, y) \in S_{2}} \lambda_{2}(u(x, y)) u(x, y) \pi_{\mathbf{u}}(x, y)-K \lambda_{1} \sum_{(x, y) \in S_{1}} \pi_{\mathbf{u}}(x, y) .
$$


The first term in (1) represents the average revenue rate collected from SUs. The second term stands for the average cost rate due to preempted SU calls. The optimal pricing policy $\mathbf{u}^{*}$ is the policy which maximizes (1) and it yields optimal profit $J^{*}$. We formulate stochastic dynamic programming (DP) problem [17] which determines the optimal pricing policy $\mathbf{u}^{*}$ of the system.

\section{B. Characteristics of the Optimal Pricing Policy}

In this section, we present a finite horizon expected discounted return DP formulation to find and characterize the optimal pricing policy. We start with some definitions:

Discounting: Our system has an exponential discount rate with parameter $\alpha \geq 0$ which implies that the reward gained in the present is more valuable than future rewards [17]. The discount rate is considered to be the rate by which the process vanishes [18].

Uniformization: Our current model is a continuous-time MDP. To convert the system to its discrete-time equivalent using uniformization, every rate coming out of a state is normalized by the maximum transition rate possible denoted $v=\lambda_{1}+\lambda_{2, \max }+C \mu+\alpha$. Without loss of generality, we set $v=1$. We scale every rate of the continuous-time MDP with $v$ which gives the probability of every transition.

Criterion: We aim to maximize the total expected discounted profit of the SP over a finite horizon. We derive the optimal pricing policy $\mathbf{u}^{*}$ which achieves this goal.

We define $n$ as the number of observation points left until the end of the time horizon. The price decision for an SU at state $(x, y)$ and time period $n$ is defined as $u$.

Definition 1: $V_{n}(x, y)$ is the maximal expected discounted profit for the system in state $(x, y)$ at time period $n$.

The finite horizon DP optimality equations are as follows:

For $n=0: V_{0}(x, y)=0 \quad$ for $\quad x, y \geq 0$

For $n \geq 1$ :

$$
\begin{aligned}
V_{n} & (x, y) \\
= & \max _{u \in \mathbb{U}}\left\{\lambda_{1} V_{n-1}(x+1, y) \mathbf{1}\{x+y<C\}\right. \\
& +\lambda_{1}\left(V_{n-1}(x+1, y-1)-K\right) \mathbf{1}\{x+y=C\} \mathbf{1}\{y>0\} \\
& +\lambda_{1} V_{n-1}(x, y) \mathbf{1}\{x+y=C\} \mathbf{1}\{y=0\} \\
& +\lambda_{2}(u)\left(V_{n-1}(x, y+1)+u\right) \mathbf{1}\{x+y<C\} \\
& +\lambda_{2}(u) V_{n-1}\left(y, c_{2}\right) \mathbf{1}\left\{c_{2}-y=0\right\} \\
& +x \mu V_{n-1}(x-1, y) \\
& +y \mu V_{n-1}(x, y-1) \\
& +\left(1-\lambda_{1}-\lambda_{2}(u)-x \mu-y \mu-\alpha\right) V_{n-1}(x, y) \\
& \times \mathbf{1}\{x+y<C\} \\
& \left.+\left(1-\lambda_{1}-C \mu-\alpha\right) V_{n-1}(x, y) \mathbf{1}\{x+y=C\}\right\} .
\end{aligned}
$$

We set $V_{n}(-1, y)=V_{n}(0, y)$ and $V_{n}(x,-1)=V_{n}(x, 0)$ when required. The value of $u$ that maximizes discounted profit is denoted $u=u_{n}^{*}(x, y)$ which is the optimal pricing decision of state $(x, y)$ at time period $n$.

Our analysis is based on the difference between two systems where the first one has one more SU than the second one. The former starts in state $(x, y+1)$ where the latter starts in state $(x, y)$ at time period $n . V_{n}(x, y+1)-V_{n}(x, y)$ is the net benefit of an additional SU when there are $n$ periods left in the horizon which is defined as the value of an additional $S U$ [16]. The next lemma states that the value of an additional $\mathrm{SU}$ is a function of $(x+y)$, i.e. total occupancy.

Lemma 1: The value of an additional $\mathrm{SU}$ at time period $n$ is a function of the total occupancy for every $(x, y)$ such that $x+y+1 \leq C$, i.e.

$$
V_{n}(x, y+1)-V_{n}(x, y)=f_{n}(x+y),
$$

where $f_{n}(\cdot)$ is recursively defined for each $n$ as:

$$
f_{n}(k)=\max _{u_{1} \in \mathbb{U}}\left\{\min _{u_{2} \in \mathbb{U}}\left\{\tilde{f}_{n}\left(k, u_{1}, u_{2}\right)\right\}\right\} \quad \text { and } \quad f_{0}(\cdot)=0,
$$

and

$\hat{f}_{n}\left(k, u_{1}, u_{2}\right)$

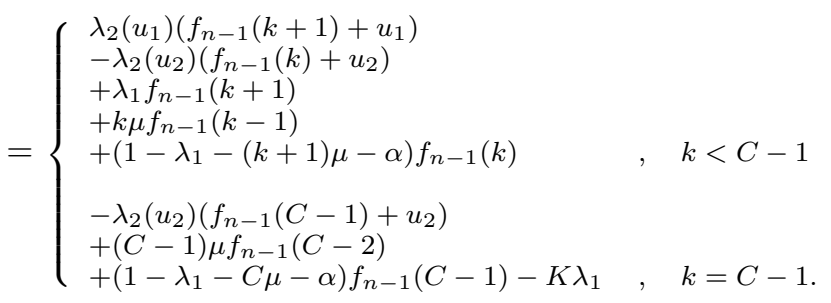

Proof: We prove this result by induction on $n$. Although the DP equations of $V_{n}(x, y)$ depend on the value of $y$ when $x+y=C$, we prove that this is not the case for the value of an additional SU. We start the induction from the end of the horizon $n=0$, i.e. $V_{0}(x, y+1)-V_{0}(x, y)=0$. Thus, Lemma 1 holds for $n=0$ by definition.

Induction step: Assume that for $n>0$, (2) holds. We show that the value of an additional $\mathrm{SU}$ at time period $n+1$ is a function of $(x+y)$ only as well, i.e.

$$
\begin{aligned}
& V_{n+1}(x, y+1)-V_{n+1}(x, y) \\
& =f_{n+1}(x+y)=\max _{u_{1} \in \mathbb{U}}\left\{\min _{u_{2} \in \mathbb{U}}\left\{\tilde{f}_{n+1}\left(x+y, u_{1}, u_{2}\right)\right\}\right\} .
\end{aligned}
$$

We need to consider two distinct cases. In the first case, $x+y<C-1$ hence, both $(x, y+1)$ and $(x, y)$ are non-preemptive states. In the second case, we consider $x+y=C-1$ where $(x, y+1)$ is a preemptive state since all channels are busy and there is at least one SU in the system. We analyze these cases separately because the corresponding DP equations are different.

Case 1. $x+y<C-1$

$$
\begin{aligned}
& V_{n+1}(x, y+1)-V_{n+1}(x, y) \\
&=\max _{u_{1} \in \mathbb{U}}\left\{\lambda_{2}\left(u_{1}\right)\left(V_{n}(x, y+2)-V_{n}(x, y+1)+u_{1}\right)\right\} \\
&+\lambda_{1} V_{n}(x+1, y+1)+x \mu V_{n}(x-1, y+1) \\
&+(y+1) \mu V_{n}(x, y) \\
&+\left(1-\lambda_{1}-x \mu-(y+1) \mu-\alpha\right) V_{n}(x, y+1) \\
&-\max _{u_{2} \in \mathbb{U}}\left\{\lambda_{2}\left(u_{2}\right)\left(V_{n}(x, y+1)-V_{n}(x, y)+u_{2}\right)\right\} \\
&-\lambda_{1} V_{n}(x+1, y)-x \mu V_{n}(x-1, y)-y \mu V_{n}(x, y-1) \\
&-\left(1-\lambda_{1}-x \mu-y \mu-\alpha\right) V_{n}(x, y) .
\end{aligned}
$$


By substituting (2) to the above expression and rearranging the terms, we obtain the following:

$$
\begin{aligned}
& V_{n+1}(x, y+1)-V_{n+1}(x, y) \\
&=\max _{u_{1} \in \mathbb{U}}\left\{\operatorname { m i n } _ { u _ { 2 } \in \mathbb { U } } \left\{\lambda_{2}\left(u_{1}\right)\left(f_{n}(x+y+1)+u_{1}\right)\right.\right. \\
&-\lambda_{2}\left(u_{2}\right)\left(f_{n}(x+y)+u_{2}\right)+\lambda_{1} f_{n}(x+y+1) \\
&+(x+y) \mu f_{n}(x+y-1) \\
&\left.\left.+\left(1-\lambda_{1}-(x+y+1) \mu-\alpha\right) f_{n}(x+y)\right\}\right\} \\
&= \max _{u_{1} \in \mathbb{U}}\left\{\min _{u_{2} \in \mathbb{U}}\left\{\tilde{f}_{n+1}\left(x+y, u_{1}, u_{2}\right)\right\}\right\}=f_{n+1}(x+y),
\end{aligned}
$$

which proves the induction for this case.

Case 2. $x+y=C-1$

$$
\begin{aligned}
& V_{n+1}(x, y+1)-V_{n+1}(x, y) \\
& =\lambda_{1}\left(V_{n}(x+1, y)-K\right)+x \mu V_{n}(x-1, y+1) \\
& \quad+(y+1) \mu V_{n}(x, y) \\
& \quad+\left(1-\lambda_{1}-x \mu-(y+1) \mu-\alpha\right) V_{n}(x, y+1) \\
& \quad-\max _{u_{2} \in \mathbb{U}}\left\{\lambda_{2}\left(u_{2}\right)\left(V_{n}(x, y+1)-V_{n}(x, y)+u_{2}\right)\right\} \\
& \quad-\lambda_{1} V_{n}(x+1, y)-x \mu V_{n}(x-1, y)-y \mu V_{n}(x, y-1) \\
& \quad-\left(1-\lambda_{1}-x \mu-y \mu-\alpha\right) V_{n}(x, y) .
\end{aligned}
$$

Similar to Case 1, we substitute (2) and rearrange the terms which results in the following expression:

$$
\begin{aligned}
& V_{n+1}(x, y+1)-V_{n+1}(x, y) \\
& =\min _{u_{2} \in \mathbb{U}}\left\{-\lambda_{2}\left(u_{2}\right)\left(f_{n}(C-1)+u_{2}\right)-K \lambda_{1}\right. \\
& \left.\quad+(C-1) \mu f_{n}(C-2)+\left(1-\lambda_{1}-C \mu-\alpha\right) f_{n}(C-1)\right\} \\
& =\min _{u_{2} \in \mathbb{U}}\left\{\tilde{f}_{n+1}\left(C-1, u_{1}, u_{2}\right)\right\}=f_{n+1}(C-1),
\end{aligned}
$$

which concludes the proof.

Combining the two cases we have examined, the induction hypothesis given in (4) is correct and we have proven that $V_{n}(x, y+1)-V_{n}(x, y)$ depends only on $(x+y)$ for all $n$.

The following theorem establishes the relationship between the optimal pricing policy and the total occupancy.

Theorem 1: The optimal price in state $(x, y)$ at time period $n$ depends only on the total number of users in the system, i.e.

$$
u_{n}^{*}(x, y)=g_{n}(x+y)
$$

where $g_{n}(\cdot)$ is recursively defined for each $n>0$ as:

$g_{n}(k)=\underset{u \in \mathbb{U}}{\operatorname{argmax}}\left\{\lambda_{2}(u)\left(f_{n}(k)+u\right)\right\} \quad$ for $\quad 0 \leq k \leq C-1$.

Proof: The optimal price $u_{n}^{*}(x, y)$ maximizes the righthand side of the DP equations. If we discard the terms that do not include the price variable $u, u_{n}^{*}(x, y)$ becomes the following:

$$
\begin{aligned}
& u_{n}^{*}(x, y) \\
& =\underset{u \in \mathbb{U}}{\operatorname{argmax}}\left\{\lambda_{2}(u)\left(V_{n-1}(x, y+1)-V_{n-1}(x, y)+u\right)\right\} .
\end{aligned}
$$

From Lemma 1, we know that $V_{n-1}(x, y+1)-$ $V_{n-1}(x, y)=f_{n}(x+y)$. When we substitute it to (7), we obtain the following result:

$$
u_{n}^{*}(x, y)=\underset{u \in \mathbb{U}}{\operatorname{argmax}}\left\{\lambda_{2}(u)\left(f_{n}(x+y)+u\right)\right\}=g_{n}(x+y) .
$$

Theorem 1 provides a drastic simplification in the determination of the optimal pricing policy. In Theorem 1, we have proven that the optimal pricing policy depends only on the total occupancy which illustrates an interesting result. The optimal pricing policy is a function of only the total occupancy although the profit function does not depend only on the total occupancy. The reason is that the optimal pricing policy is not determined by the profit function itself; rather it depends on the value of an additional SU.

So far, we have proven our results for the finite horizon discounted profit to use induction on $n$. Since our system satisfies the standard conditions given in [19], all conclusions apply to the infinite horizon $\alpha$-discounted case by taking the limit $n \rightarrow \infty$. Furthermore, the average profit can be computed considering the case $\alpha \rightarrow 0$.

\section{Infinite Horizon Average Return DP Formulation of the Simplified System}

Now that we have shown that the optimal pricing policy depends only on the total occupancy, we formulate an infinite horizon average return problem for additional results. We cannot reduce our original model to a one-dimensional (1D) MDP since the profit function does not depend only on the total occupancy. Instead of using the original system, we utilize an auxiliary system in the infinite horizon average return formulation of the original system. The model of the auxiliary system is the same as the original system with one exception: the system imposes a punishment $K$ when all channels are busy and a PU arrival occurs, regardless of the presence of SUs, i.e. the auxiliary system is exactly the same as the original system other than the fact that a cost $K$ occurs if a PU gets blocked because of other PUs in the system. The profit function and optimal pricing policy depend only on the total occupancy and it has the same optimal pricing policy as the original system.

Let $Q_{\mathbf{u}}$ denote the average profit rate of the auxiliary system under policy $\mathbf{u}$. The relationship between the profit functions $Q_{\mathbf{u}}$ and $J_{\mathbf{u}}$ is given by:

$$
J_{\mathbf{u}}=Q_{\mathbf{u}}+K \lambda_{1} E\left(\lambda_{1} / \mu, C\right) .
$$

$E\left(\lambda_{1} / \mu, C\right)$ is the blocking probability of a PU when there are no SU arrivals, which corresponds to the Erlang-B formula:

$$
E\left(\lambda_{1} / \mu, C\right)=\frac{\frac{\left(\lambda_{1} / \mu\right)^{C}}{C !}}{\sum_{n=0}^{C} \frac{\left(\lambda_{1} / \mu\right)^{n}}{n !}} .
$$

Thus, for any policy $\mathbf{u}, J_{\mathbf{u}}$ and $Q_{\mathbf{u}}$ differ by the constant $K \lambda_{1} E\left(\lambda_{1} / \mu, C\right)$. Consequently, the policy that maximizes $Q_{\mathbf{u}}$ is the same as the policy that maximizes $J_{\mathbf{u}}$ in the average return case. 


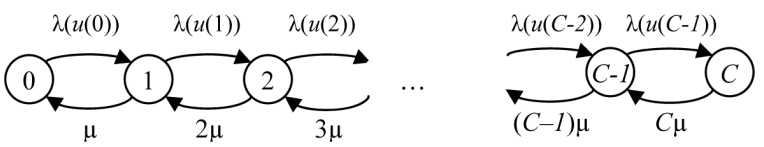

Fig. 1. 1D continuous-time MDP representation of the auxiliary system

For the simplified model which considers the total occupancy to determine the optimal policy, we define new system parameters. Let $0 \leq i \leq C$ denote the occupancy levels of the auxiliary system which is the sum of PUs and SUs in the system, i.e. $i=x+y$. Our system parameters are the same as before: We still have Poisson arrivals and exponentially distributed call durations with mean $\mu^{-1}$. Thus, we still consider a continuous-time birth-death Markov Process. The only modification is that we replace the definition of state $(x, y)$ with $i$. Prices are chosen from the discrete set $\mathbb{U}$ which is defined earlier. Price advertised to SUs at the total occupancy level $i$ is $u(i)$. The arrival rate of SUs is a function of the price denoted by $\lambda_{2}(u(i))$. Then, the total arrival rate to any state $i$ is $\lambda(u(i))=\lambda_{1}+\lambda_{2}(u(i))$.

Next, we provide an average return DP formulation of the auxiliary system using Bellman's equations [17] in order to obtain the optimal price vector $\mathbf{u}^{*} \triangleq$ $\left(u^{*}(0), u^{*}(1), \ldots, u^{*}(C-1)\right)$, which provides the optimal price at each occupancy level of the original system as well.

We model the auxiliary system as a 1D MDP where the total occupancies are considered as the states, which is illustrated in Fig. 1. $Q_{\mathbf{u}}$, the average profit rate of the auxiliary system under policy $\mathbf{u}$, is as follows:

$$
Q_{\mathbf{u}}=\sum_{i=0}^{C-1} \lambda_{2}(u(i)) u(i) \pi_{\mathbf{u}}(i)-K \lambda_{1} \pi_{\mathbf{u}}(C) .
$$

Under the same optimal pricing policy, the relationship between the optimal average profit functions $Q^{*}$ and $J^{*}$ is unchanged and given in (8).

Next, we formulate the average return DP problem for the auxiliary system. The use of Bellman's equations is possible in this system, since all the states in the Markov chain are recurrent [17]. We need convert the continuous-time Markov chain to its discrete-time equivalent using uniformization. We normalize every rate coming out of each state by the maximum rate possible, which is $v^{\prime}=\lambda_{1}+\lambda_{2, \max }+C \mu$. Without loss of generality, we set $v^{\prime}=1$. The corresponding Bellman's equations are as follows:

$$
\begin{aligned}
Q^{*}+h(i)= & \max _{u \in \mathbb{U}}\left[\lambda_{2}(u) u+h(i+1) \lambda(u)+h(i-1) i \mu\right. \\
& +h(i)(1-\lambda u-i \mu)] \text { for } i=0,1, \ldots, C-1 \\
Q^{*}+h(C)= & -\lambda_{1} K+h(C-1) C \mu+h(C)(1-C \mu) .
\end{aligned}
$$

The optimal prices and the optimal average profit are found by solving the given Bellman's equations. We set $h(C)=0$, so $h(i)$ is the relative reward of state $i$ with respect to $h(C)$. Then, $h(i)$ is the average expected difference of the rewards of two processes where the former starts from state $i$ and the latter starts from state $C$.

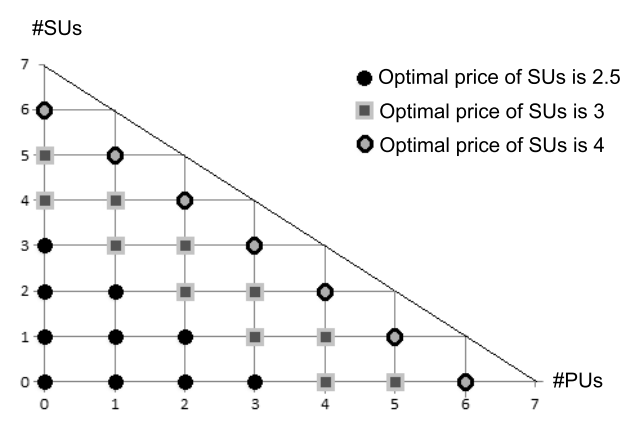

Fig. 2. The optimal pricing policy of SUs in the system of Example 1, with inelastic PUs. The advertised price is constant on diagonal states on which the total occupancy is the same.

\section{Monotonicity Properties of the Optimal Pricing Policy}

In this section, we emphasize some monotonicity properties of the simplified system. The proofs of these properties follow similar lines as [4] and [20]. The main differences are that our system is preemptive and has a different cost structure.

The following two lemmas denote that the relative reward is a decreasing and concave function of the total occupancy.

Lemma 2: As total occupancy increases, the value of relative reward decreases i.e., $h(i+1)-h(i) \leq 0$ for $0 \leq i<C$.

Lemma 3: The relative rewards are concave in occupancy i.e., $h(i)-h(i-1) \geq h(i+1)-h(i)$ for $0<i<C$.

Lemmas 2 and 3 lead to the next theorem on the relationship between occupancy and the optimal pricing policy.

Theorem 2: As total occupancy increases, optimal price increases as well i.e., $u^{*}(i+1) \geq u^{*}(i)$ for $0 \leq i<C-1$.

Now that we have specified the properties of the optimal pricing policy, we present an example to illustrate the results of Theorem 1 and Theorem 2.

Example 1: In this example, we set $C=7, \mu=1, \lambda_{1}=$ $3, K=10, \Delta u=0.5, \mathbb{U}=[0,4]$ and the demand function is $\lambda_{2}(u)=(4-u)_{+}$where $(\cdot)_{+}=\max (\cdot, 0)$. The resulting optimal pricing policy is illustrated in Figure 2.

We observe that the states with the same occupancy have the same optimal pricing decision. Furthermore, the optimal prices increase with the total occupancy.

\section{SPECIAL CASES}

In this section, we examine some special cases of the optimal pricing policy we have characterized.

\section{A. Optimal Admission Control Policy of SUs}

In admission control, the SP either accepts a user upon arrival or rejects it by following an admission control policy a. If an SU call is accepted, a constant reward of $R>0$ is charged per call and the corresponding arrival rate is $\lambda_{2}(R)$. If a call is rejected, the SP sets the price to $u_{\max }$, hence the arrival rate of SUs $\lambda_{2}\left(u_{\max }\right)$ drops to 0 . We consider admission control as a special case of pricing which includes two possible prices in the control set. There is a 


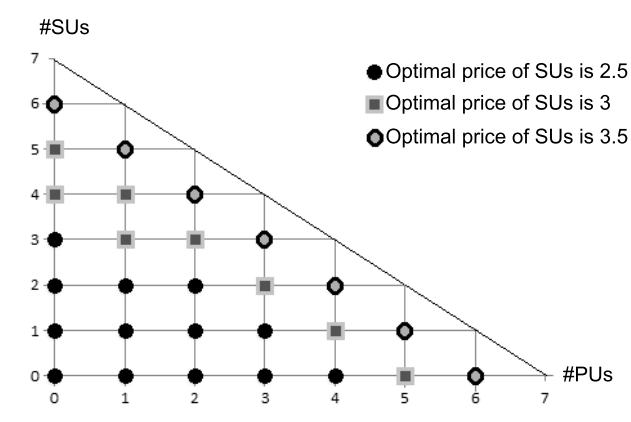

Fig. 3. The optimal pricing policy of SUs in the system of Example 2, with elastic PUs. The advertised price is not constant on the diagonal states.

mapping between the pricing decision and admission control decision at state $(x, y)$. We define a new control parameter $a(x, y)$ which is a restricted binary version of $u(x, y)$ (i.e. $a(x, y) \in\{0,1\}) . a(x, y)$ is the admission control decision of an arriving $\mathrm{SU}$ when the current state is $(x, y)$.

The following corollary applies theorems 1 and 2 to the optimal admission control problem.

Corollary 1: The optimal admission control policy $\mathbf{a}^{*}$ of SUs is of threshold type and it depends only on the total number of users in the system. Thus, there exists an optimal occupancy threshold $T^{*}$ for a system at state $(x, y)$ such that $0 \leq x+y<T^{*}$. If the total occupancy is less than $T^{*}$, an incoming SU call is accepted. Otherwise, it is rejected.

Note that, the very same result is obtained in Theorem 1 of [21] using a different analysis method. Hence, we have corroborated our findings using two different methods.

\section{B. Discussion of the Optimal Pricing with Elastic PUs}

Assume that every definition and parameter in the original system model stays the same except the PU arrival rates. In the original model, PUs have inelastic Poisson arrival rates with mean $\lambda_{1}$. We consider a variant of the original system where the PUs are elastic to price and a price $\hat{u}(x, y)$ is advertised to PUs at state $(x, y)$. Furthermore, the PUs have a demand function $\lambda_{1}(\hat{u}(x, y))$, which follows the assumptions 1 and 2. We would like to determine the optimal pricing policies of both PUs and SUs to maximize the overall profit by solving a joint maximization problem. The next example demonstrates that Theorem 1 does not apply to the optimal pricing policy of SUs.

Example 2: Set $C=7, \mu=1, K=7, \Delta u=0.5, \Delta \hat{u}=$ 1 and the demand functions for PUs and SUs are $\lambda_{1}(\hat{u})=$ $(10-\hat{u})_{+}$and $\lambda_{2}(u)=(4-u)_{+}$. The resulting optimal pricing policy is demonstrated in Figure 3.

Notice that Theorem 1 does not apply to the optimal pricing policies of SUs. For instance, for $x+y=4$, we have different optimal pricing decisions for different states.

\section{CONCLUSIONS}

In this paper, we analyze the profit maximization problem of preemptive systems. Inelastic PUs and elastic SUs coexist in a system where PUs have preemptive priority over SUs.
We propose a 2D Markov chain model and formulate the average profit rate. Our main contribution is to analytically prove that the optimal pricing policy depends only on the total occupancy. Our results provide a simplification in determination of the optimal pricing not only in CR networks but also for any preemptive system, such as wireless medium access control protocols, Multi-Protocol Label Switching networks and multitasking in operating systems [22].

\section{REFERENCES}

[1] Ericsson, "Ericsson predicts mobile data traffic to grow 10-fold by 2016." http: / /www. ericsson. com/news/1561267, 2011.

[2] J. Peha, "Approaches to spectrum sharing," Communications Magazine, IEEE, vol. 43, no. 2, pp. 10-12, 2005.

[3] P. Tortelier, S. Grimoud, P. Cordier, S. B. Jemaa, L. Iacobelli, C. Le Martret, J. van de Beek, S. Subramani, W. H. Chin, M. Sooriyabandara, L. Gavrilovska, V. Atanasovski, P. Mahonen, and J. Riihijarvi, "Flexible and spectrum aware radio access through measurements and modelling in cognitive radio systems," Faramir Tech. Rep. 248351, Faramir Project, 2010.

[4] I. Paschalidis and J. Tsitsiklis, "Congestion-dependent pricing of network services," IEEE/ACM Transactions on Networking, vol. 8, no. 2, pp. 171-184, 2000.

[5] H. Mutlu, M. Alanyali, and D. Starobinski, "Spot pricing of secondary spectrum usage in wireless cellular networks," in In proceedings of IEEE INFOCOM, pp. 682-690, 2008.

[6] N. Gans and S. Savin, "Pricing and capacity rationing for rentals with uncertain durations," Management Science, vol. 53, pp. 390-407, 2007.

[7] P. Dube, V. S. Borkar, and D. Manjunath, "Differential join prices for parallel queues: Social optimality, dynamic pricing algorithms and application to internet pricing," in In Proceedings of IEEE INFOCOM, pp. 276-283, 2002.

[8] B. Miller, "A queueing reward system with several customer classes," Management Science, vol. 16, pp. 234-245, 1971.

[9] R. Ramjee, D. Towsley, and R. Nagarajan, "On optimal call admission control in cellular networks," Wireless Networks, vol. 3, pp. 29-41, 1996.

[10] E. Ormeci, A. Burnetas, and J. van der Wal, "Admission policies for a two class loss system," Stochastic Models, vol. 17, pp. 513-540, 2001.

[11] W. Helly, "Two doctrines for the handling of two-priority traffic by a group of n servers," Operations Research, vol. 10, no. 2, pp. 268-269, 1962.

[12] J. Garay and I. Gopal, "Call preemption in communication networks," in In Proceedings of IEEE INFOCOM, vol. 3, pp. 1043-1050, 1992.

[13] S. Xu and J. Shanthikumar, "Optimal expulsion control - a dual approach to admission control of an ordered-entry system," Operations Research, vol. 41, pp. 1137-1152, 1993.

[14] A. Brouns and J. van der Wal, "Optimal threshold policies in a two-class preemptive priority queue with admission and termination control," Queueing Systems, vol. 54, pp. 21-33, 2006.

[15] A. Brouns, Queueing models with admission and termination control Monotonicity and threshold results. $\mathrm{PhD}$ thesis, Eindhoven University of Technology, 2003.

[16] M. Ulukus, R. Gullu, and L. Ormeci, "Admission and termination control of a two class loss system," Stochastic Models, vol. 27, no. 1, pp. 2-25, 2011.

[17] D. Bertsekas, Dynamic Programming and Optimal Control. Athena Scientific, third ed., 2007.

[18] J. Walrand, An Introduction to Queueing Networks. Prentice Hall, 1988.

[19] S. Ross, Introduction to Stochastic Dynamic Programming. Academic Press, 1983.

[20] H. Mutlu, Spot Pricing of Secondary Access to Wireless Spectrum. PhD thesis, Boston University, 2010.

[21] A. Turhan, M. Alanyali, and D. Starobinski, "Optimal admission control of secondary users in preemptive cognitive radio networks," in IEEE International Symposium on Modeling and Optimization in Mobile, Ad Hoc, and Wireless Networks, WiOpt 2012, pp. 138-144, 2012.

[22] Z. Zhao, S. Weber, and J. Oliveira, "Preemption rates for a parallel link loss network," Performance Evaluation, vol. 66, no. 1, pp. 21-46, 2006. 